\title{
Fabrication of micromachined pipettes in a flow channel for single molecule handling of DNA
}

\author{
Cristina Rusu, Ronny van't Oever, Meint de Boer, Henri Jansen, Erwin Berenschot, \\ M.L. Bennink", J.S. Kanger*, B.G. de Grooth*, Miko Elwenspoek and J. Greve* \\ A. van den Berg ( $\mu$ TAS), J. Brugger (NanoLink) \\ Electrical Engineering, Transducers Technology Group \\ "Applied Physics, Biophysical Techniques Group \\ University of Twente, MESA Research Insitute, P.O. Bos 217, 7500 AE Enschede, the Netherlands, \\ c.r.rusu@tn.utwente.nl, phone: +31-53-489 3161, fax: +31-53-4891105
}

\begin{abstract}
We have developed a micromachined flow cell consisting of a flow channel integrated with micropipettes. The flow cell is used in combination with an optical trap set-up (optical tweezers) to study mechanical and structural properties of $\lambda$ DNA molecules. The flow cell was realised using silicon micromachining including the so-called buried channel technology to fabricate the micropipettes, the wet etching of glass to create the flow channel, and the powder blasting of glass to make the fluid connections. The volume of the flow cell is $2 \mu 1$. The pipettes have a length of $130 \mu \mathrm{m}$, a width of $5-10 \mu \mathrm{m}$, a round opening of $1 \mu \mathrm{m}$ and can be processed with different shapes. Using this flow cell we stretched single molecules ( $\lambda$-DNA) showing typical force-extension curves also found with conventional techniques. These pipettes can be also used for drug delivery, for injection of small gas bubbles into a liquid flow to monitor the streamlines, and for the mixing of two liquids to study diffusion effects. The paper describes the design, the fabrication and testing of the flow cell.
\end{abstract}

\section{INTRODUCTION}

There are various ways to study mechanical and structural properties of single DNA molecules [1]. One of these methods uses an optical trap in conjunction with two polystyrene beads to apply a stretching force on a DNA molecule. Each end of the single DNA molecule is biochemically bound to a bead using streptavidin-biotin. One bead is held by a capillary glass pipette and the other bead by an optical trap, which is used to apply and measure the stretching force on the DNA molecule at a certain elongation $[2,3]$.

The assemble of the bead-DNA-bead system before the stretching experiment can start is, briefly, as follows. Through the flow channel a buffer with beads is flown. A bead is captured with the optical tweezers (fig. 1a) and is transferred on top of the pipette (1b). A second bead is trapped by the optical tweezers (1c). Another buffer with DNA is flown through the channel (1d) until one molecule sticks to the bead in the optical trap (1e). The fluid flow is used to connect the second bead to the DNA molecule (1f). After this procedure, the stretching experiment can start $(1 \mathrm{~g})$.
Although this technique reveals nicely some typical characteristics, such as the spring constant of the molecule, experiments on an assembly of DNA molecules have to be performed one-by-one. Having many micropipettes in one flow channel, parallel measurements on single molecules may be performed allowing for rapid 'statistical' data acquisition.

This paper describes a way, i.e. silicon micromachining, to integrate many pipettes in the same flow channel to allow for the just mentioned simultaneous measurements.

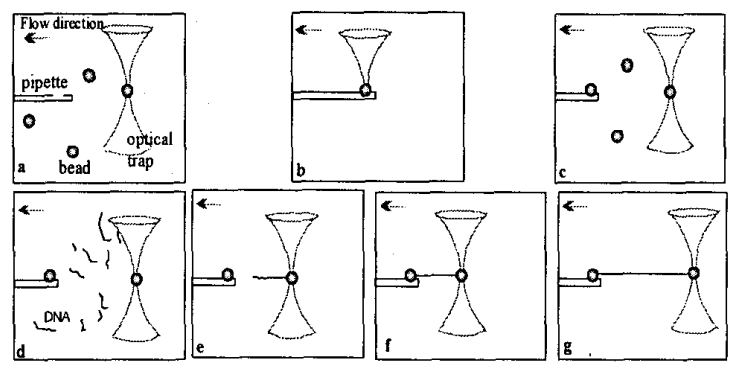

Fig. 1. Sketch of the attachment of a single DNA between two polystyrene beads, one gripped with a glass pipette and one with the optical tweezers. 


\section{SET-UP FOR DNA HANDLING}

The current optical trap set-up (fig. 2) is a home-made microscope system and a flow cell unit that are described in more details in [4]. A laser diode is used for the optical tweezers [5], in which micrometer-sized polystyrene beaqds can be trapped just behind the focal point of a 100x objective (water immersion, NA=1.2). When external forces are exerted on the trapped bead, the bead will move out of the centre of the laser beam. The transparent bead acts as a small lens and causes a deflection of the beam proportional to the force [6]. This is measured by a quadrant detector. A maximum force of $70 \mathrm{pN}$ can be exerted on the bead, the optical trap stiffness is about $200 \mathrm{pN} / \mu \mathrm{m}$, and the precision in determining the DNA molecule length is $5-10 \mathrm{~nm}$ [4].

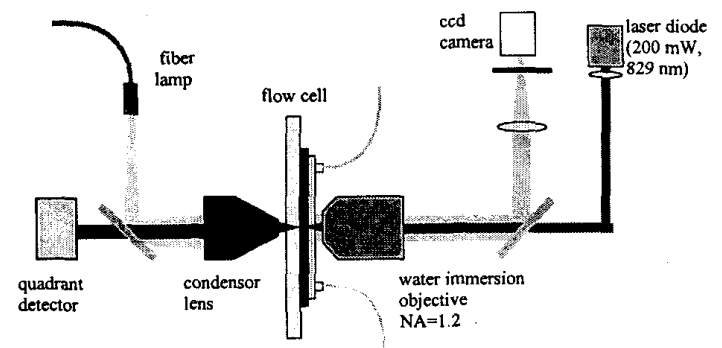

Figure 2. Sketch of the optical trap set-up with flow cell.

\section{DESIGN RULES FOR THE FLOW CELL}

The constraints resulting from the optical trap set-up give the design rules for the flow cell.

(i) The flow cell has to be optically transparent. The channel is etched in silicon, and Pyrex is chosen as a cover glass material to allow the light to pass.

(ii) The thickness of the cover glass must match the objective to create a good optical trap inside the flow channel. In our case $175 \mu \mathrm{m}$.

(iii) The position of the micropipettes in the flow channel is determined by the maximum depth of the optical trap to the cover glass.

(iv) The micropipette opening is defined by the bead diameter, smaller than $2 \mu \mathrm{m}$.

(v) Small sample volume, ca. $2 \mu$ l.

\section{MICROMACHINING THE FLOW CELL}

The general design of a micromachined flow cell is given in fig. 3. The micropipettes are made of silicon nitride based on the buried channel technology (BCT) [7]. The fabrication of the flow channel in both Pyrex and silicon is based on bulk micromachining. A powder blasting process [8] of Pyrex is used to enable the fluid connection for the flow channel and the micropipettes. The two wafers will be anodically bonded. The fluid interconnection between the flow cell and the set-up holder uses a packing material made from a dry film resist layer.

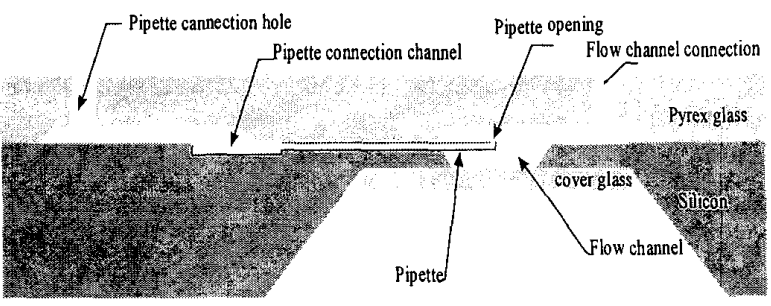

Fig. 3. Cross section of a flow cell consisting of integrated micropipette in a flow channel.

\section{Pipette fabrication}

Figure 4 gives the outline of the basic steps of the buried channel technology. A $1 \mu \mathrm{m}$ wide trench, that is made in silicon by Reactive Ion Etching (RIE) (step a) is coated with a protection layer (thermal oxide) (step b). The layer is removed from the bottom of the trench by RIE (step c), and the buried structure is made by an isotropic etch of the trench (step d). Then the protection layer is removed and the structure is made by growing in the trench a layer of LPCVD silicon nitride (step e). Next the surrounding silicon is etched away in $\mathrm{KOH}$ resulting in a micropipette (step f). Due to the BCT process, the opening of the pipette will be on the top side of the pipette.

The direct integration of the micropipettes in the flow channel is possible due to the fact that BCT is a singlewafer process, is self-aligned and uses one-sided processing without the need of complicated assembling techniques.
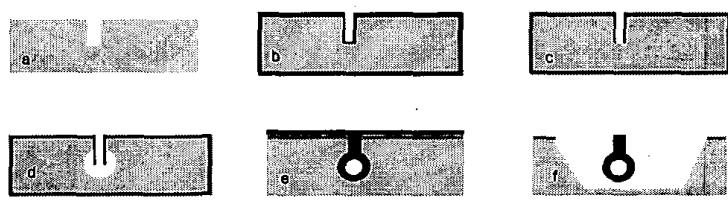

Fig. 4. Process outline of a micropipette using BCT (crosssection).

\section{Different micropipette shapes}

Based on the principle of BCT four different shaped pipettes have been processed (fig. 5).

- Shape 1 can be made by an isotropic under etch of a slit etched in a silicon oxide mask (fig. 5-1). A disadvantage of this shape is that when a bead with a DNA molecule is hold on top of the hole, the DNA may stick to the flat top of the pipette (fig. 6). A larger distance between the pipette opening, where the bead is captured, and the pipette surface would be preferable.

- Shape 2 can be achieved by an isotropic under etch of the trench done by RIE (fig. $4 \mathrm{~d}$, fig. 7) or by a wet etching. A problems with a wet etching process can be the wetting of the trench. Advantage of the isotropic etch is that the etched result is independent of the crystal orientation of the silicon with respect to the mask layout. 


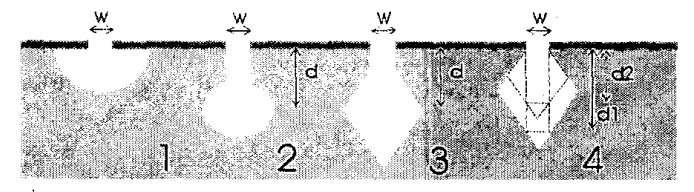

Fig. 5. Four different shapes for pipettes in silicon possible with $B C T$.

- When $\mathrm{KOH}$ etch solution is used (fig. 5-3), the walls of the channel will be very smooth (silicon [111] planes). Disadvantage is that the obtained result depends on the crystal orientation of the silicon.

- Shape 4 also makes use of $\mathrm{KOH}$ (fig. 5-4, fig. 8) has the advantage that the size of the channel easily can be regulated by the trench depth, and there is no need for bottom etching of the trench. Disadvantage is again that the obtained result depends on crystal orientation.

In shape 2 and 3 the depth of the structure underneath the surface can be tuned by the depth (d) of the trench (fig. 4c). The trench depth is limited by the width (w) of the trench and by the maximum aspect ratio that can be achieved during RIE etching [7]. The other limit to the trench depth is the easiness in removing the trench bottom.

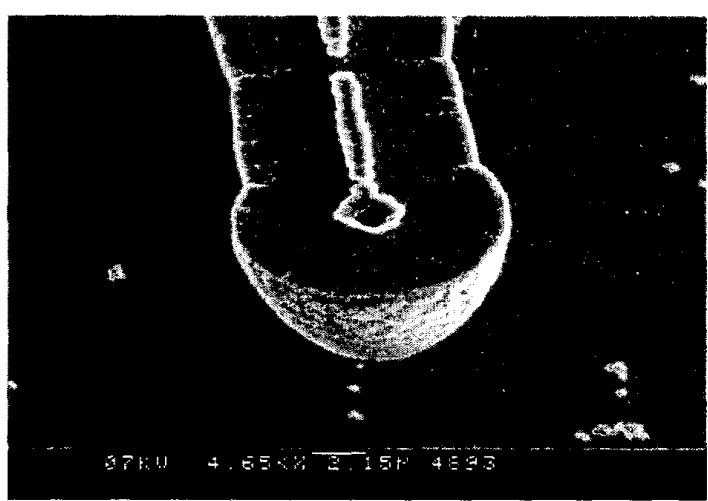

Fig. 6. Micropipette of shape 1 (opening l $\mu \mathrm{m}$ ).

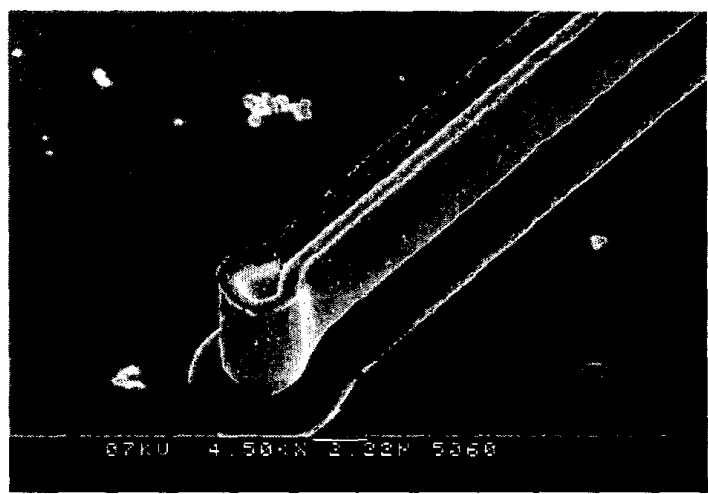

Fig. 7. Micropipette of shape 2, using mask layout I (opening I $\mu \mathrm{am}$ ).

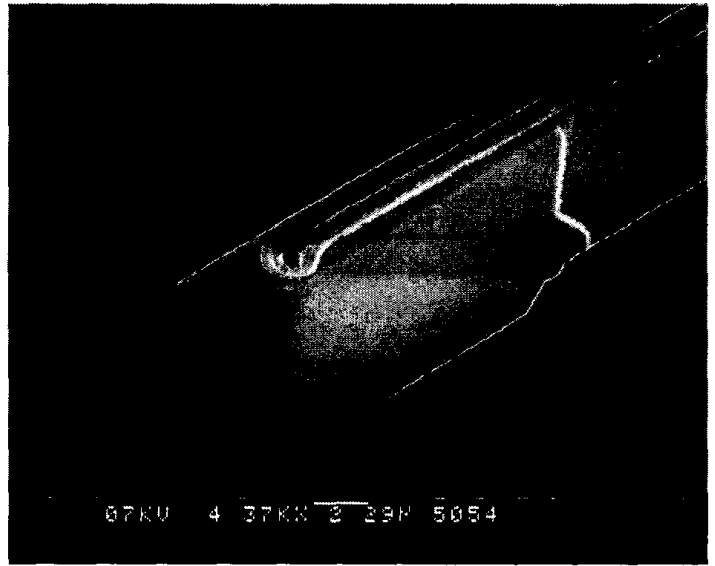

Fig. 8. Micropipette of shape 4.

\section{Integration of micropipette opening}

The mask layouts shown in fig. 10 are used to integrate an opening for the pipette. All the process steps from fig. 4 step a until step e are done with this mask. Crosssections in direction of $\mathrm{B}, \mathrm{C}, \mathrm{D}$, and $\mathrm{A}$, are shown in fig. 11 and fig. 12, respectively. The circular opening in the mask layout of type $I$ is deformed by the influence of the trench as can be seen in fig. 7. Due to this artefact, a second mask layout, type II, has been integrated. As can be observed in fig. 9, the mask layout type II gives better results for the pipette opening definition since the opening is separated from the trench.

The connection of the pipette opening to the pipette tube with mask type I is automatically done when the trench is etched (step a in fig. 4). For the mask type II, until step $c$, the two parts of the pipette are still separated. Due to the under etching of the trench in step $d$ the two parts will connect (fig. 9 and fig. 12).

Many micropipettes are simultaneously integrated in a flow channel of $100 \mu \mathrm{m}$ height, $500 \mu \mathrm{m}$ wide and $3 \mathrm{~cm}$ long (fig. 13). This small channel volume, $2 \mu$, requires only very small sample volume. The micropipettes shown have a length of ca. $130 \mu \mathrm{m}$, width of 5-10 $\mu \mathrm{m}$, and a circular-like opening of $1 \mu \mathrm{m}$.

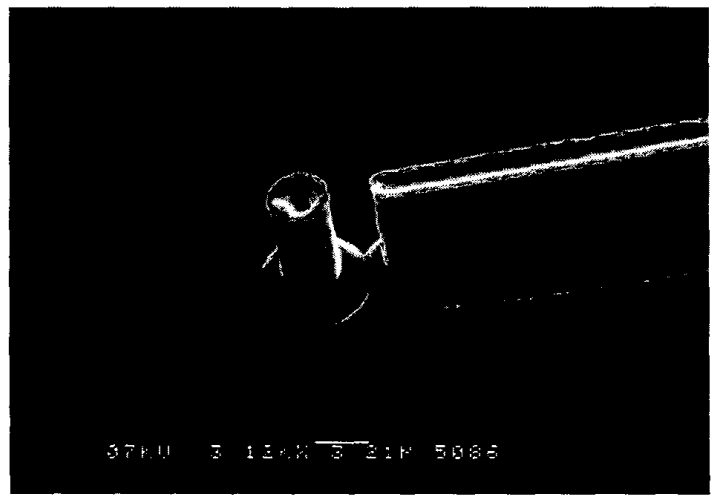

Fig. 9. Micropipette of shape 2, using mask layout II to achieve a more circular-like opening. 


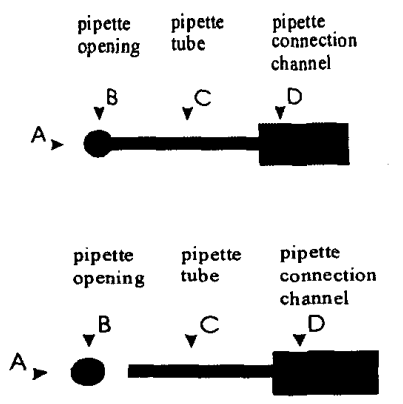

Fig. 10. Two different mask layouts (top view) to integrate an opening in the pipettes made by $B C T$.

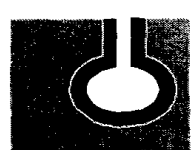

B

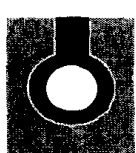

C

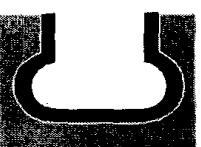

D
Fig. 11. Cross section in the $B, C$, and D plane of the buried channel.

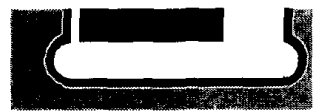

mask type I

mask type I

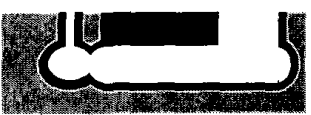

mask type II

mask type II
Fig. 12. Integration of the micropipette opening: cross section of the pipette in direction of $A$ using the two mask layouts.

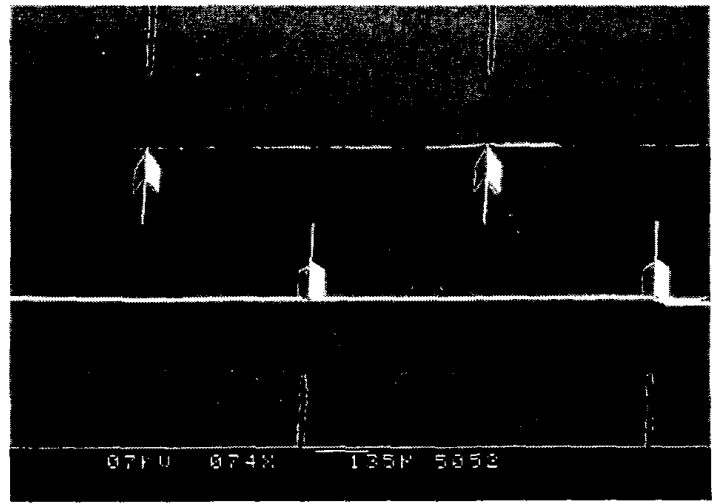

Fig. 13. Several micropipettes on their silicon support above the surface.

\section{Flow channel}

From fig. 3 it can be seen that the micropipettes connections and the upper part of the flow channel are made in Pyrex glass. The Pyrex wafer is patterned and etched in $50 \% \mathrm{HF}$ solution by using a $1 \mu \mathrm{m}$ sputtered silicon layer as mask.

The next step is the powder blasting through the Pyrex wafer creating the $300 \mu \mathrm{m}$ diameter connection holes [8]. For this, a layer of dry film resist (Ordyl BF405) is deposited on the other side of the Pyrex wafer (not the structured side) and patterned with photolithography. Then, the Pyrex wafer is diced, the sputtered Si mask is removed by $\mathrm{KOH}$ and the flow channel side is anodically bonded to the silicon wafer in order to assemble the flow cell.

\section{Holder and device connection}

The fluidic connections between the flow cell and a holder uses a new packing material instead of a macroscopic connection (like a Viton O-ring causing a large dead volume). This packing material is, again, the dry film resist (Ordyl BF405). The dry film is patterned with photolithography and developed in $0.2 \% \mathrm{Na}_{2} \mathrm{CO}_{3}$ solution. After drying, the film is applied onto the flow cell and heated to $100^{\circ} \mathrm{C}$ for a good adhesion. When the flow cell is mounted into the holder, a simultaneously clamping and heating of the Ordyl is done for a tight connection. A schematic representation of the connection between the flow cell and holder is shown in fig. 14 .

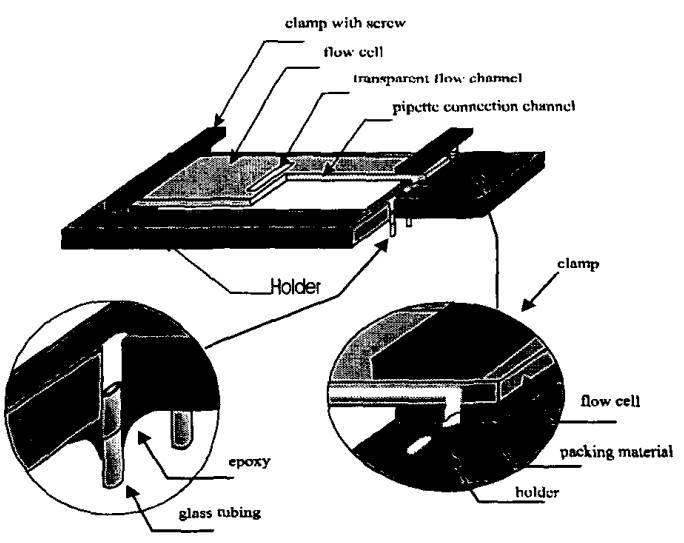

Fig. 14. Overview of the holder with details of connections. 


\section{RESULTS AND DISCUSSIONS}

Tests, performed with shape 2 micropipettes, have shown that the pipettes are open (Fig.15). As can be seen the micropipettes can be used to locally inject small air bubbles into a fluid flow.

From the first experiments with the polystyrene beads and micropipettes in a flow channel it could be seen that the beads were sticking to the silicon nitride. Fig. 16 shows a lot of beads sticking to the micropipette. The strength of the sticking force is such that the beads could not be released with the optical tweezers $(\sim 70 \mathrm{pN})$.

The beads on the pipette could easily be removed when the flow cell was washed with $1 \%$ SDS (sodium dodecyl sulfate) solution or with BSA (bovine serum albumin). Figure 17 shows a micropipette and a bead that is gripped by the optical tweezers. Then, the bead is transferred to the pipette opening and gripped, fig. 18 .

In fig. 19a, the DNA molecule [6] is held in between the beads, and the force sensor marker shows zero force. Of course, the DNA itself can not be seen using optical wavelength due to its extreme small diameter (2nm). By moving the pipette away from the optical trap the DNA molecule is stretched, fig. $19 \mathrm{~b}$.

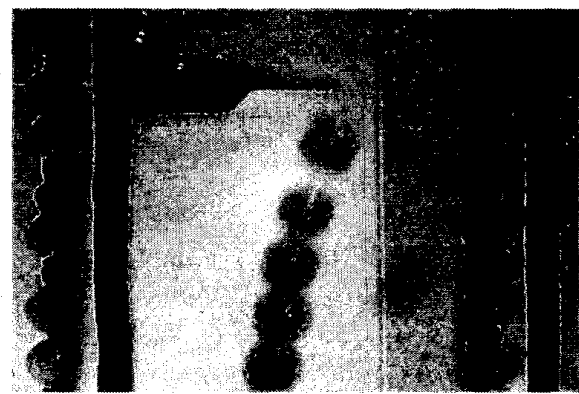

Fig. 15. Making air bubbles with a silicon nitride micropipette in a flow channel.

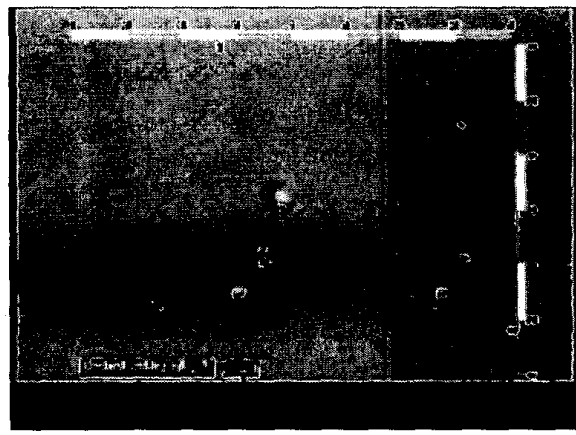

Fig. 16. Sticking of polystyrene beads on a silicon nitride micropipette.

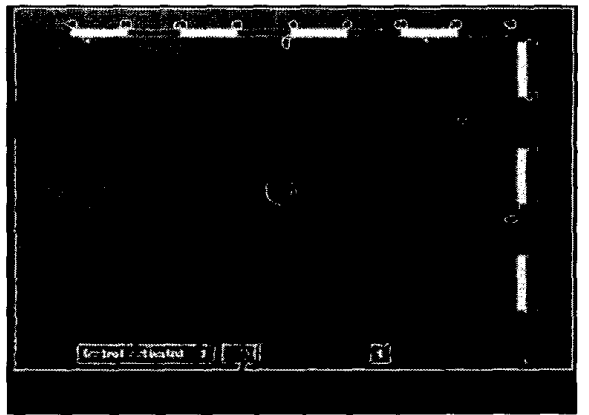

Fig. 17. A 2 fm polystyrene bead hold by the optical tweezers next to the micropipette.

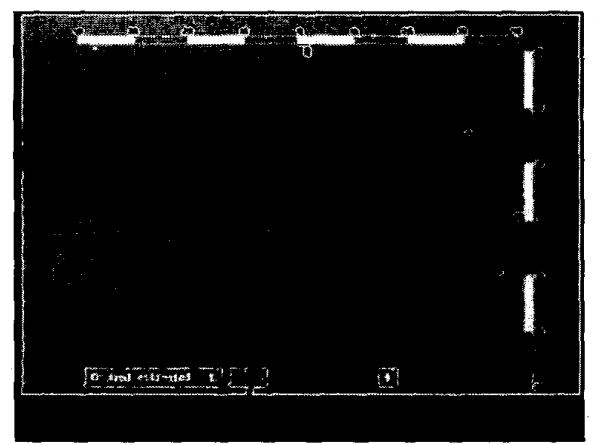

Fig. 18. A bead gripped by the micropipette.

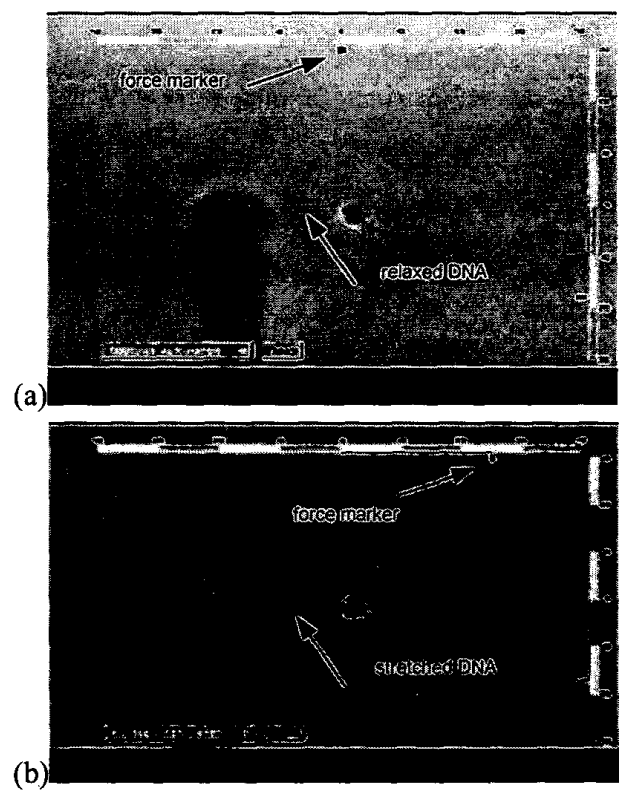

Fig. 19. Two beads gripped on a micromachined pipette and by the optical tweezers having a DNA molecule (not observable) hold in between; (a) no force applied;(b) $30 p N$ force applied. 


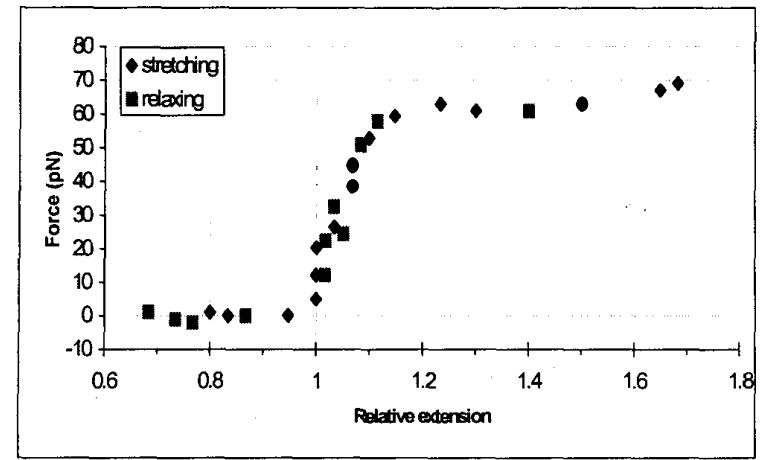

Fig. 20. Experimental stretch measurements with the micromachined flow cell and the micropipettes.

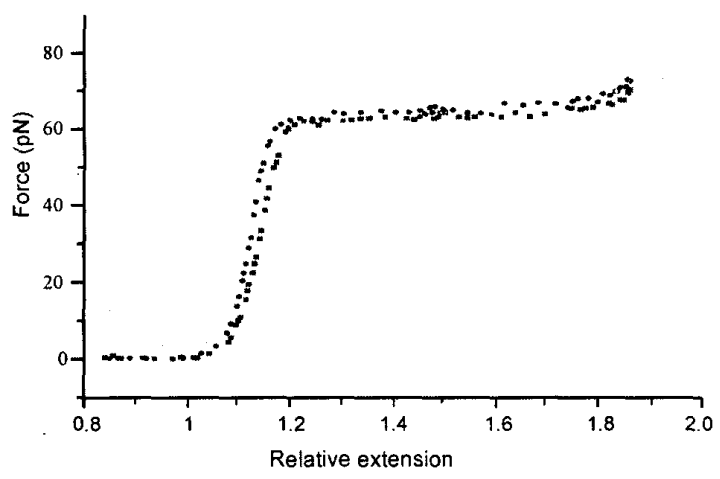

Fig. 21. Experimental stretch measurements with the conventional flow cell and the capillary pipettes [4].

The force-extension curve obtained with the micromachined flow cell and micropipettes, fig. 20, shows a similar behaviour as the typical one (fig. 21) [4]. There are two curves. The diamond points represent the stretching of the DNA, and the square points are measured during the relaxation of the same molecule.

The behaviour of the single molecule up to a few $\mathrm{pN}$ is the entropic elasticity as described by [Smith]. As soon the contour length of DNA is reached (relative extension $\mathrm{L} / \mathrm{L}_{0}=1$ ) the force increases very fast up to about $60 \mathrm{pN}$. For $\mathrm{L} / \mathrm{L}_{0}$ between 1 and 1.1 the DNA shows a spring-like behaviour. Above this level the DNA molecule is stretching out within a force of only a few $\mathrm{pN}$.

An effect was observed when the optical trap was brought within $5 \mu \mathrm{m}$ from the pipette: the light from the laser was deflected by the pipette. The light of the laser beam diverges rapidly below the bead given an incorrect force measurement.
The present micropipettes (ca. $12 \mu \mathrm{m}$ high, $5-10 \mu \mathrm{m}$ width) are suitable for the study of the mechanical properties of long DNA molecules $(>10 \mu \mathrm{m})$.

For chemical study of DNA, shorter DNA molecule $(5 \mu \mathrm{m})$ has to be used. For this investigation, the micropipettes have to be downscaled. A smaller pipette (diameter $2-3 \mu \mathrm{m}$ ) can be constructed by etching a less deep trench with a smaller width $(<1 \mu \mathrm{m})$ and reducing the etch time of the isotropic etch (fig. 4d).

\section{CONCLUSIONS}

We have demonstrated the possibility to integrate micropipettes into a flow channel to study mechanical and structural properties of DNA molecules. Moreover, various shapes of the micropipettes have been fabricated having the convenience of choosing for each type of experiment the best pipette shape and size. The conventional flow cell with glass capillary pipettes have been replaced successfully by this micromachined flow cell. Force-extension curves from both the conventional and micromachined technology were found to be in excellent agreement. For chemical study of DNA, the micropipette should be downscaled.

\section{References}

[1] Special issue of Science, vol. 283, 1999.

[2] S.B. Smith, Y. Cui, C. Bustamante, Overstretching BDNA: the elastic response of individual double-stranded and single-stranded DNA molecules, Science, vol. 271, p. 795-799, 1996.

[3] P. Cluzel, A. Lebrun, C. Heller, R. Lavery, J. Viovy, D. Chatenay, F. Caron, DNA: an extensible molecule, Science, vol. 271, p.792-794, 1996.

[4] M.L. Bennink, O.D. Schärer, R. Kanaar, K. SakataSogawa, J.M. Schins, J.S. Kanger, B.G. de Grooth, J. Greve, Single molecule manipulation of double-stranded DNA using optical tweezers, Cytometry, vol. 36 , p. 200 208, 1999.

[5] A. Ashkin, J.M. Dziedzic, J.E. Bjorkholm, S. Chu, Observation of a single-beam gradient force optical trap for dielectric particles, Optics Letters, vol. 11(5), p.288$290,1986$.

[6] L.P. Ghislain, N.A. Switz, W.W. Webb, Measurement of small forces using an optical trap, Rev. Sci. Instrum., 65(9), p.2762-2767, 1994.

[7] M.J. de Boer, H.V. Jansen, W. Tjerkstra, M.C. Elwenspoek, Micromachining of Buried Micro Channels in silicon, submitted to JMEMS, 1999.

[8] H. Wensink, J.W. Berenschot, H.V. Jansen, M.C. Elwenspoek, Micromachining by powder blasting, This proceeding. 